See discussions, stats, and author profiles for this publication at: https://www.researchgate.net/publication/262859770

\title{
Texture analysis of the media-layer of the left and right common carotid artery
}

Conference Paper · June 2014

DOI: 10.1109/BHI.2014.6864456

CITATION

6 authors, including:

Christos P Loizou

University of Cyprus

155 PUBLICATIONS 1,811 CITATIONS

SEE PROFILE

Maura Griffin

The Vascular Noninvasive Diagnostic Centre London

201 PUBLICATIONS 5,114 CITATIONS

SEE PROFILE

Some of the authors of this publication are also working on these related projects:

Project mEducator View project

Project eENERCA - European Network for Rare and Congenital Anaemias View project
READS

128

Niki Georgiou

36 PUBLICATIONS 792 CITATIONS

SEE PROFILE

Efthyvoulos C Kyriacou

Frederick University

174 PUBLICATIONS 2,436 CITATIONS

SEE PROFILE 


\title{
Texture Analysis of the Media-Layer of the Left and Right Common Carotid Artery
}

\author{
Christos P. Loizou-IEEE Senior Member, Niki Georgiou, Maura Griffin, Eftyvoulos Kyriacou-IEEE \\ Senior Member, Andrew Nicolaides, and Constantinos S. Pattichis-IEEE Senior Member
}

\begin{abstract}
The intima-media thickness (IMT) of the common carotid artery (CCA) is a well-known indicator of cardiovascular disease (CVD). The objective of this study was to investigate the application of texture analysis of the medial layer (ML) of the CCA, and how texture features vary between the left and right carotid sides, as well as how these are affected by CVD. The study was performed on 200 longitudinal-section ultrasound images from 150 normal subjects, and 50 symptomatic subjects suffering with CVD. The ML was segmented automatically by a snakes segmentation system and 61 different texture features were extracted. This study showed that only the gray level difference statistics (GLDS) correlation measure of the ML component could be used to differentiate between normal subjects, and subjects suffering with CVD for either the left or the right CCA sides. We furthermore found that a number of texture features were significantly different between the left and the right $\mathrm{CCA}$ sides. We have found no other studies in the literature where these findings could be compared to. These findings should be further validated on a larger number of subjects as well as combined with additional risk factors.
\end{abstract}

\section{INTRODUCTION}

Cardiovascular disease (CVD) is the largest cause of death worldwide [1]. Atherosclerosis is the main reason leading to CVD and can result to heart attack, and stroke [1], [2]. Carotid intima-media-thickness (IMT), is a measurement of the thickness of the innermost two layers of the arterial wall of the common carotid artery (CCA) [2], and it is well accepted as a validated surrogate marker for atherosclerotic disease. It was also proposed in [3], that the media layer (ML) (see also Fig. 1) thickness and textural characteristics, may be investigated for evaluating the risk that a subject might have to develop a stroke. The objective of this study was to investigate the application of texture analysis of ML of the CCA, and how texture features vary between the left and right carotid sides, and how these are affected by CVD.

There is only one study reported in the literature [3], where texture changes of the ML were investigated and a few other studies [4]-[6], where texture changes of the intima-

Christos P. Loizou is with the department of Computer science, at Intercollege, Limassol, Cyprus. Phone: +35725381180 ; fax: +35725386982 (e-mail: loizou.c@ lim.intercollege.ac.cy;panloicy@logosnet.cy.net).

Efthyvoulos Kyriacou is is with the department of Computer Science at the Frederick University, Nicosia, Cyprus (e-mail: ekyriac@ucy.ac.cy).

Constantinos S. Pattichis is with the department of Computer Science at the University of Cyprus, Nicosia, Cyprus (e-mail: pattichi@cs.ucy.ac.cy).

Niki Georgiou, Maura Griffin and Andrew Nicolaides are with the Vascular screening and diagnostic center, Nicosia, Cyprus (e-mail: anicolaides1@gmail.com). media complex (IMC) (but not for the ML alone were investigated). It was also recently shown in [7] that the IMT is not equal in both CCA sides, but no statistical significant differences were found. It was found in [7] that the IMT in the left CCA side is slightly larger than that of the right CCA side.

It is noted that most IMT studies in the past were performed on individuals at the age of 50 years or more [8] [11], thus excluding the possibility to reveal the differences at an earlier stage of the disease [8]. It was also shown in [10], that the CCA IMT may be possibly used for the prediction of possible infarct side and for the prediction of potential risk of stroke by evaluating the IMT on both sides of the CCA [12]. In [13], the side differences in CCA IMT measurements, and their prognostic value in patients with stable coronary artery disease were evaluated. The study in [13] showed that the left and the right CCA may exhibit different prognostic values in the investigated population.

IMT can be measured through the segmentation of the IMC, which corresponds to the intima and media layers of the arterial wall. There is a plethora of ultrasound imaging CCA IMC segmentation algorithms which were reviewed in [14]. In two recent studies performed by our group [15], [16], we presented a semi-automatic method for the IMC segmentation [15]. These systems use active contour models in a normalized rectangular region of interest where speckle removal was applied [17]. In [16], we presented an extension of the integrated system proposed in [15], where also the intima layer and the ML of the CCA were segmented. Furthermore, a fully automated method based on active contours was recently proposed for the segmentation of the IMC from ultrasound images of the CCA [18].

The objective of this study was to investigate whether textural characteristics extracted from the ML of the CCA, segmented automatically by a snake's segmentation system [15], [16] vary between the left and right carotid sides, and how these are affected by CVD.

\section{Materials \& Methods}

\section{A. Recording and Preprocessing of Ultrasound Images}

A total of $200 \mathrm{~B}$-mode longitudinal ultrasound images of the CCA which display the arterial wall as a regular pattern (see Fig. 1a and b) that correlates with anatomical layers were recorded. The images were acquired by the ATL HDI-5000 ultrasound scanner (Advanced Technology Laboratories, 
Seattle, USA) [19] with a resolution of 576X768 pixels with 256 gray levels from 105 men and 95

women with a mean \pm SD age of $65.57 \pm 10.61$ years. For the recordings, a linear probe (L74) at a recording frequency of $7 \mathrm{MHz}$ was used. Assuming a sound velocity propagation of $1550 \mathrm{~m} / \mathrm{s}$ and 1 cycle per pulse, we thus have an effective spatial pulse width of $0.22 \mathrm{~mm}$ with an axial system resolution of $0.11 \mathrm{~mm}$ [19]. We used bicubic spline interpolation to resize all images to a standard pixel density of 16.66 pixels $/ \mathrm{mm}$ (with a resulting pixel width of 0.06

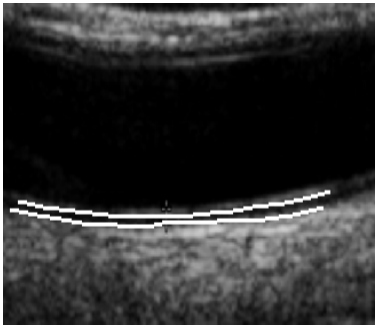

(a) Left CCA ML

(c)

Fig. 1. Automated ML segmentation of the a) left side ( $\mathrm{MLT}_{\text {mean }}=$ $0.39 \mathrm{~mm}, \mathrm{MLT}_{\max }=0.45 \mathrm{~mm}, \mathrm{MLT}_{\min }=0.30 \mathrm{~mm}, \mathrm{MT}_{\text {median }}=0.35$ $\mathrm{mm})$, and b) right side $\left(\mathrm{MLT}_{\text {mean }}=0.33 \mathrm{~mm}, \mathrm{MLT}_{\max }=0.45 \mathrm{~mm}\right.$, $\mathrm{MLT}_{\min }=0.29 \mathrm{~mm}, \mathrm{MLT}_{\text {median }}=0.31 \mathrm{~mm}$ ), respectively. c) and d) segmented ML componenets of the left and right sides respectively.

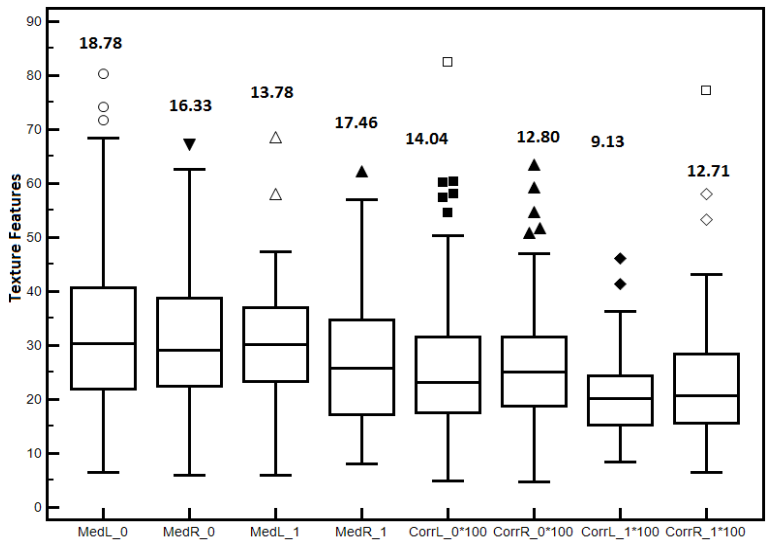

Fig. 2. Box plots for the left (L) and right (R) sides of the CCA ML for the texture features Median and Correlationx100 for $\mathrm{CVD}=\mathrm{NO}(0)$ and $\mathrm{CVD}=\mathrm{YES}(1)$ respectively. Inter-quartile range values are shown above the box plots. Straight lines connect the nearest observations with 1.5 of the inter-quartile range (IQR) of the lower and upper quartiles. Unfilled rectangles indicate possible outliers with values beyond the ends of the 1.5xIQR.

$\mathrm{mm})$. The images were recorded at a cohort study in a Cyprus mountain village from asymptomatic, apparently healthy subjects $(\mathrm{N}=150)$, and symptomatic subjects, which have already developed CVD events $(\mathrm{N}=50)$. The ages of the two groups (with and without CVD) were statistically significant different ( $\mathrm{p}=0.004$, using the Mann-Whitney rank sum test). A written informed consent was obtained according to the instructions of the local ethics committee.
Brightness adjustments of ultrasound images were carried out in this study based on the method introduced in [20]. In this study the linear scaling filter (despeckle filter-DsFlsmv) [17], utilizing the mean and the variance of a pixel neighborhood was used to filter the ultrasound images prior to segmentation and texture analysis.

\section{B. Automatic ML and IMC Snake Segmentations}

The IMC and ML regions were automatically segmented using the automated snakes segmentation system proposed and evaluated on 100 ultrasound images of the CCA in [15] and [16], which is based on the Williams \& Shah [21] snake segmentation method. The extracted snake contours (see Fig. 1a and $1 \mathrm{~b}$ ), correspond to the adventitia and media borders of the ML. The distance is computed between the two boundaries, at all points along the arterial wall segment of interest moving perpendicularly between pixel pairs, and then averaged to obtain the mean MT thickness ( $\left.\mathrm{MLT}_{\text {mean }}\right)$.

\section{Texture Analysis}

A total of 61 different texture features were extracted from the automated ML segmented regions of interest, where only the most significant are presented as follows: (i) Statistical Features [22]: a) mean, b) variance, c) median value, d) skewness, e) kurtosis, f) energy and g) entropy. (ii) Spatial Gray Level Dependence Matrices (SGLDM) as proposed by Haralick et al. [23]: a) angular second moment (ASM), b) contrast, c) correlation, d) sum of squares variance, e) inverse difference moment, f) sum average, g) sum variance, h) sum entropy, i) entropy, j) difference variance, k) difference entropy, and 1) information measures of correlation (IMOC). For a chosen distance $d$ (in this work $\mathrm{d}=1$ was used) and for angles $\theta=0^{\circ}, 45^{\circ}, 90^{\circ}$, and $135^{\circ}$, we computed four values for each of the above texture measures. (iii) Gray Level Difference Statistics (GLDS) [24]: a) homogeneity, b) contrast, c) energy, d) entropy, e) mean, and f) and l) information measures of correlation (IMOC). The above features were calculated for displacements $\delta=(0,1),(1,1),(1,0),(1,-1)$, where $\delta \equiv(\Delta x, \Delta y)$, and their mean values were taken. (iv) Neighborhood Gray Tone Difference Matrix (NGTDM) [25]: a) coarseness, b) contrast, c) busyness, d) complexity, and e) strength. (v) Statistical Feature Matrix (SFM) [26]: a) coarseness, b) contrast, c) periodicity, and d) roughness. (vi) Laws Texture Energy Measures (LTEM) [26]: LL-texture energy from LL kernel, EE-texture energy from EE-kernel, SS-texture energy from SS-kernel, LE-average texture energy from LE and EL kernels, ES-average texture energy from ES and SE kernels, and LS-average texture energy from LS and SL kernels. (vii) Fractal Dimension Texture Analysis (FDTA) [26]: The Hurst coefficients for dimensions 4, 3 and 2 were computed. (viii) Fourier Power Spectrum (FPS) [26]: a) radial sum, and b) angular sum. 
TABLE I

TEXTURE FEATURE ANALYSIS (MEDIAN ( $($ IQR)) OF THE ML OF THE LEFT AND THE RIGHT CCA USING AUTOMATED SEGMENTATIONS FOR SUBJECTS THAT HAD NOT DEVELOPED CVD (CVD=NO, N=150) AND SUBJECTS THAT HAD DEVELOPED A CVD EVENT (CVD=YES, N=150) (-/-). THE THIRD COLUMN SHOWS THE RESULTS OF MANN-WHITNEY RANK SUM TEST (AT $\mathrm{p}<0.05$ ) BETWEEN LEFT CCA AND RIGHT CCA FOR CVD=NO / CVD=YES ON SELECTED FEATURES. SIMILARLY, THE LAST COLUMN SHOWS THE RESULTS OF THE SPEARMAN CORRELATION COEFFICIENT, $\rho$.

\begin{tabular}{|c|c|c|c|c|}
\hline Feature & $\begin{array}{c}\text { Left CCA } \\
\mathrm{CVD}=\mathrm{NO} / \mathrm{CVD}=\mathrm{YES}\end{array}$ & $\begin{array}{c}\text { Right CCA } \\
\text { CVD=NO } / \text { CVD }=\text { YES }\end{array}$ & $\begin{array}{c}\text { MANN-WHITNEY } \\
\text { CVD=NO / CVD=YES }\end{array}$ & $\stackrel{\rho}{C V D=N O} / \mathrm{CVD}=\mathrm{YES}$ \\
\hline \multicolumn{5}{|c|}{ First Order Statistics (FOS) } \\
\hline Median & $30.39(18.1) / 30.87(17.9)$ & $29.15(16.19) / 28.31(19.22)$ & $\mathrm{NS} / \mathrm{NS}$ & $0.23 / 0.32$ \\
\hline \multicolumn{5}{|c|}{ Spatial Gray Level Dependence Matrices (SGLDM) } \\
\hline $\mathrm{ASM}^{*} 1000$ & $2.3(1.82) / 2.1(1.6)$ & $2.1(1.62) / 3.4(2.6)$ & $\mathrm{S} / \mathrm{S}$ & $0.24 / 0.22$ \\
\hline Entropy & $6.39(0.70) / 6.51(0.67)$ & $6.35(0.65) / 6.28(0.63)$ & $\mathrm{S} / \mathrm{S}$ & $0.23 / 0.15$ \\
\hline IMOC & $0.35(0.071) / 0.37(0.066)$ & $0.36(0.082) / 0.35(0.072)$ & $\mathrm{S} / \mathrm{S}$ & $0.18 / 0.09$ \\
\hline \multicolumn{5}{|c|}{ Gray Level Difference Statistics (GLDS) } \\
\hline Entropy & $0.65(0.2) / 0.62(0.25)$ & $0.61(0.25) / 0.64(0.24)$ & $\mathrm{S} / \mathrm{S}$ & $0.15 / 0.22$ \\
\hline Correlation & $0.23(0.14) / 0.20(0.08)$ & $0.25(0.12) / 0.21(0.13)$ & $\mathrm{S} / \mathrm{S}$ & $0.22 / 0.33$ \\
\hline Sum Average & $1.77(1.21) / 2.0(1.19)$ & $1.81(1.3) / 1.59(1.35)$ & $\mathrm{NS} / \mathrm{S}$ & $0.16 / 0.17$ \\
\hline IMOC & $0.19(0.06) / 0.18(0.06)$ & $0.18(0.08) /(0.18(0.08)$ & $\mathrm{S} / \mathrm{S}$ & $0.36 / 0.19$ \\
\hline \multicolumn{5}{|c|}{ Neighborhood Gray Tone Difference Matrix (NGTDM) } \\
\hline Contrast & $0.21(0.14) / 0.22(0.1)$ & $0.22(0.16) / 0.22(0.13)$ & $\mathrm{S} / \mathrm{S}$ & $0.045 / 0.13$ \\
\hline Busyness & $4.6(9.5) / 2.99(5.76)$ & $5.1(8.7) / 4.36(1.06)$ & $\mathrm{S} / \mathrm{S}$ & $0.29 / 0.22$ \\
\hline \multicolumn{5}{|c|}{ Laws Texture Energy Measures (LTEM) } \\
\hline $\mathrm{EE}$ & $673(287) / 820(3.99)$ & $728(3.83) / 667(293)$ & $\mathrm{S} / \mathrm{NS}$ & $0.12 / 0.13$ \\
\hline ES-SE & $207(82) / 215(133)$ & $226(111) / 195(101)$ & $\mathrm{S} / \mathrm{NS}$ & $0.21 / 0.29$ \\
\hline \multicolumn{5}{|c|}{ Fractal Dimension (FD) } \\
\hline $\mathrm{H} 1$ & $0.41(0.09) / 0.39(0.1)$ & $0.39(0.1) / 0.43(0.1)$ & $\mathrm{S} / \mathrm{S}$ & $0.34 / 0.29$ \\
\hline \multicolumn{5}{|c|}{ Intima-media and Media Thickness Measurements } \\
\hline IMT [mm] & $0.67 \pm 0.21 / 0.81 \pm 0.26$ & $0.63 \pm 0.17 / 0.78 \pm 0.21$ & NS / NS & $0.73 / 0.69$ \\
\hline $\mathrm{MT}[\mathrm{mm}]$ & $0.29 \pm 0.09 / 0.35 \pm 0.12$ & $0.27 \pm 0.09 / 0.31 \pm 0.12$ & NS / NS & $0.67 / 0.78$ \\
\hline
\end{tabular}

IQR: Inter-Quartile-Range, ASM: Angular second moment, IMOC: Information measures of correlation, H1 Hurst coefficients, EE: EE-texture Energy form EE-kernel, ES-SE: ES-average texture energy from ES and SE kernels. S: Significantly different at $\mathrm{p}<0.05$, NS: non-significantly different at $\mathrm{p} \geq 0.05$.

\section{RESULTS}

Figure 1 illustrates an example of automated ML segmentations acquired from a female CVD subject at the age of 75 years. We show in Fig. 1a) the left and in Fig. 1b) the right ML segmentations respectively.

Table I presents the median and inter quartile range $( \pm \mathrm{IQR})$ of different texture features for the ML between the left and the right $\mathrm{CCA}$ for $\mathrm{CVD}=\mathrm{NO}$ and $\mathrm{CVD}=\mathrm{YES}$. All features tabulated except the median showed significant difference between the left and the right sides, either for subjects with $\mathrm{CVD}=\mathrm{No}$, or subjects with $\mathrm{CVD}=$ Yes. Moreover, rather low correlation coefficients were obtained between the left and the right sides for both $\mathrm{CVD}=\mathrm{No}$, and $\mathrm{CVD}=$ Yes subjects. The last two rows present the IMT and MT measurements as well. Figure 2 presents box plots for the features median (FOS feature group) and correlation (GLDS feature group) for both sides for $\mathrm{CVD}=\mathrm{No}$ and $\mathrm{CVD}=$ Yes.

Table II presents the ML texture features that showed significant difference between the $\mathrm{CVD}=\mathrm{No}$ and $\mathrm{CVD}=\mathrm{Yes}$ groups. Only GLDS Correlation could differentiate between the two groups for both sides. Correlation is a measure of gray-tone linear dependencies and of heterogeneity. Heterogeneous images have higher correlation values.

\section{CONCLUDING REMARKS}

This study showed that only the GLDS Correlation measure of the ML component could be used to differentiate between normal subjects, and subjects suffering with CVD for either the left or the right CCA sides. We have found no other studies in the literature where these findings could be 
TABLE II

MANN WHITNEY RANK SUM TEST RESULTS AT $(\mathrm{P}<0.05)$ ON ML TEXTURE FEATURES OF THE LEFT CCA AND THE RIGHT CCA FOR CVD $=$ NO VERSUS $C V D=$ YES SUBJECTS. ONLY THE FEATURES THAT SHOWED SIGNIFICANT DIFFERENCE ARE PRESENTED (ALSO SHOWN IN BOLD IN TABLE I).

\begin{tabular}{|l|c|c|}
\hline \multicolumn{1}{|c|}{ Feature } & \multicolumn{2}{c|}{ CVD=NO vs CVD=YES } \\
\hline \multicolumn{3}{|c|}{ Left CCA Level Difference Statistics (GLDS) } \\
\hline Correlation & 0.04 & 0.03 \\
\hline Sum Average & - & 0.04 \\
\hline \multicolumn{3}{|c|}{ Laws texture Energy Measures (LTEM) } \\
\hline EE- & 0.02 & - \\
\hline ES-SE & 0.02 & \\
\hline
\end{tabular}

compared to. Exception is the study of [3], where it was shown that ML texture features may be used to separate subjects with age groups.

Some limitations of the proposed method include the presence of acoustic shadowing together with strong speckle noise, which hinders the visual and automatic analysis in ultrasound imaging. Such images, with bad visual perception, were neither included in this study nor were they delineated by the experts [15]-[8], [14], [18], [20]. We have also excluded from our segmentation experiments images with extensive echolucency and calcification. In the present study in less than $3 \%$ of the cases the positioning of the initial snake contour was not calculated correctly. Furthermore, only vessels without atherosclerotic plaques were segmented in this study.

The findings of this study should be further validated on a larger sample, a task which is currently undertaken by our group. Additional risk factors, such as age, sex, weight, blood pressure and others, should be taken into account for predicting future cardiovascular outcomes. Future work will investigate whether it is possible to identify a group of patients at risk of atherosclerosis based on their texture features extracted from the ML of high-resolution ultrasound images of the CCA.

\section{REFERENCES}

[1] S. Mendis, P. Puska, B. Norrving, "Global Atlas on cardiovascular disease prevention and control," WHO, 2011.

[2] P. Touboul, M. Hennerici, S. Meairs, H. Adams, et al., "Mannheim carotid intima-media thickness consensus (2004-2006)," Cerebr. Diseases, vol. 23, pp. 75-80, 2007.

[3] C.P. Loizou, M. Pantziaris, M.S. Pattichis, E. Kyriakou, C.S. Pattichis, "Ultrasound image texture analysis of the intima and media layers of the common carotid artery and its correlation with age and gender," Comput. Med. Imag. Graph., vol. 33, no.4, pp. 317-324, 2009.

[4] S.M. Ellis, P.S. Sidhu, "Granularity of the carotid artery intimamedial layer: reproducibility of quantification by a computer-based program,” BJOR, vol. 37, pp. 595-600, 2000.

[5] F. Bartolomucci, M. Paterni, C. Morizzo, M. Kozakova, et al. "Early structural changes of carotid artery in familial hypercholesterolemia," J. Clin. Hypertens., vol. 14, pp. 125A-126A, 2001.

[6] L. Lind, J. Andersson, M. Roenn, T. Gustavsson, "The echogenecity of the intima-media complex in the common carotid artery is closely related to the echogenecity in plaques," Atherosclerosis, vol. 195, pp. 411-14, 2007.

[7] C.P. Loizou, C.S. Pattichis, N. Georghiou, M. Griffin, A.N. Nicolaides, "A comparison of ultrasound intima-media thickness measurements of the left and right common carotid artery," $12^{\text {th }}$ Int.
Conf. Bioinf. \& Bioeng. (BIBE), Crete, Nov. 11-13, pages 4, T.1.5.4, 2013.

[8] Y. Arbel, N. Maharshak, A. Gal-Oz, I, Sapira, et al., "Lack of difference in the intimal medial thickness between the left and the right carotid arteries in the young," Acta. Neurol. Scand., vol. 115, pp. 409-412, 2007.

[9] S. A. Rodriguez Hernandez, A. A. Kroon, M. P. van Boxtel et al., "Is there a side predilection for cerebrovascular disease?," Hypertension, vol. 42, pp. 56-60, 2003.

[10] O. Onbas, M. Kantarci, A. Okur, U. Bayraktutan, A. Edis, N. Ceviz, "Carotid intima-media thickness: is it correlated with stroke side?," Acta. Neurol. Scand., vol. 111, pp. 169-171, 2005.

[11] Y. Sun, C. H. Lin, C. J. Lu, P. K. et al., "Carotid atherosclerosis, intima media thickness and risk factors-an analysis of 1781 asymptomatic subjects in Taiwan," Atheroscl., vol. 164, pp. 89-94, 2002.

[12] X. Luo, Y. Yang, T. Cao, Z. Li, "Differences in left and right carotid artery intima media thickens and the associated risk factors," Clinic. Radiol., vol. 66, pp. 393-398, 2011.

[13] S. W-L. Lee Kong, J. J. Siu, S. L. Han Hai, Y.-M. Lam, et al., "Side differences of carotid intima-media thickness in predicting cardiovascular events among patients with coronary artery disease," Angiol., vol. 62, no. 3, pp. 231-236, 2010.

[14] F. Molinari, G. Zeng, J. S. Suri, "A state of the art review on intimamedia thickness measurement and wall segmentation techniques for carotid ultrasound," Comp. Meth. and Progr. in Biomed., vol. 100, pp. 201-221, 2010.

[15] C. P. Loizou, C. S. Pattichis, M. Pantziaris, T. Tyllis, A. N. Nicolaides, "Snakes based segmentation of the common carotid artery intima media," Med. Biolog. Eng. Comput., vol. 45, pp. 35-49, 2007.

[16] C. P. Loizou, C. S. Pattichis, A. N. Nicolaides, M. Pantziaris, "Manual and automated media and intima thickness measurements of the common carotid artery," IEEE Trans. Ultras. Ferroel. Freq. Contr., vol. 56, no. 5, pp. 983-994, 2009.

[17] C. P. Loizou, C. S. Pattichis, C. I. Christodoulou, R. S. H. Istepanian, M. Pantziaris, A. N. Nicolaides, "Comparative evaluation of despeckle filtering in ultrasound imaging of the carotid artery," IEEE Trans. Ultras. Ferroel. Freq. Contr., vol. 52, no. 10, pp. 1653-1669, 2005.

[18] S. Petroudi, C.P. Loizou, M. Pantziaris, C.S. Pattichis, "Segmentation of the common carotid intima-media complex in ultrasound images using active contours," IEEE Trans. Biomed. Eng., vol. 59, no. 11, pp. 3060-3069, Nov. 2012.

[19] A Philips Medical System Company, "Comparison of image clarity, SonoCT real-time compound imaging versus conventional 2D ultrasound imaging," ATL Ultrasound, Report, 2001.

[20] T. Elatrozy, A. N. Nicolaides, T. Tegos, A. Zarka, M. Griffin, M. Sabetai, "The effect of B-mode ultrasonic image standardization of the echodensity of symptomatic and asymptomatic carotid bifurcation plaque," Int. Angiol., vol. 17, no. 3, pp. 179-186, 1998.

[21] D. J. Williams, M. Shah, "A fast algorithm for active contour and curvature estimation," Comp. Vis. Image Underst., vol. 55, no. 1, pp. 4-26, 1992.

[22] C.I. Christodoulou, C.S. Pattichis, M. Pantziaris, A.N. Nicolaides "Texture-based classification of atherosclerotic carotid plaques," IEEE Trans. Med. Imag., vol. 22, no. 7, pp. 902-912, 2003.

[23] R.M. Haralick, K. Shanmugam, I. Dinstein, "Texture features for image classification," IEEE Trans. Syst. Man. Cyber., SMC-3:61021, 1973.

[24] J.S. Weszka, C.R. Dyer, A. Rosenfield, "A comparative study of texture measures for terrain classification," IEEE Trans. Syst. Man. Cyber., SMC-6, pp.269-85, 1976.

[25] M. Amadasun, R. King, "Textural features corresponding to textural properties," IEEE Trans Syst. Man. Cyber., vol. 19, no. 5, pp. 264741989.

[26] C.M. Wu, Y.C. Chen, K.-S Hsieh, "Texture features for classification of ultrasonic images," IEEE Trans. Med. Imag., vol. 11, pp. 141-52, 1992. 